\title{
Regularity criteria for the topology of algebraic curves and surfaces
}

\author{
L. Alberti \& B. Mourrain \\ GALAAD, INRIA, \\ BP 93, 06902 Sophia Antipolis \\ France
}

\begin{abstract}
In this paper, we consider the problem of analysing the shape of an object defined by polynomial equations in a domain. We describe regularity criteria which allow us to determine the topology of the implicit object in a box from information on the boundary of this box. Such criteria are given for planar and space algebraic curves and for algebraic surfaces. These tests are used in subdivision methods in order to produce a polygonal approximation of the algebraic curves or surfaces, even if it contains singular points. We exploit the representation of polynomials in Bernstein basis to check these criteria and to compute the intersection of edges or facets of the box with these curves or surfaces. Our treatment of singularities exploits results from singularity theory such as an explicit Whitney stratification or the local conic structure around singularities. A few examples illustrate the behavior of the algorithms.
\end{abstract}

\section{Introduction}

In this paper, we consider the problem of analysing the shape of an object defined by polynomial equations on a bounded domain. Such a problem appears naturally when one has to compute with (algebraic) implicit surfaces [6], but also in algorithms on parameterised curves and surfaces. Typically computing the intersection of two parameterised surfaces leads to the problem of describing or analysing an implicit curve in a 4-dimensional space $[23,18]$.

Our aim is to describe subdivision methods, which given input equations defining such an implicit object, compute a linear approximation of this object, with the same topology. The field of application of such methods is Geometric Modeling, where the (semi)-algebraic models used to represent shapes are considered approximations of the real geometry. That is, either their coefficients are known with some error or the model itself is an approximation of the actual geometry. In this modelisation process, it is assumed that making the error tend to 0 , the representation converges to the actual geometry, at least conceptually. We are going to follow this line, with two specific objectives in mind:

- provide guarantees if possible.

- adapt the computation to the local difficulties of the problem. 
Several methods exist to visualize or to mesh a (smooth) implicit surface. Ray tracing techniques [26] which compute the intersection between the ray from the eye of the observer and the first object of the scene, produce very nice static views of these surfaces. However isolated singular curves are not well treated and the output of such methods is an image, not a mesh that can be used for other computation.

The famous "marching cube" method [30,44] developed in order to reconstruct images in 3 dimensions starting from medical data, is based on the construction of grids of values for the function and of sign analysis. It is not adaptive to the geometry of the shape, gives no guarantee of correctness and applies only to smooth surfaces.

Marching polygonizer methods improve the adaptivity of the marching cube by computing only the "useful" cells $[4,5,24]$, that is those which cut the surface. The algorithm starts from a valid cube (or tetrahedron), and propagate towards the connected cells, which cut the surface. Other variants of the Marching Cube approach have been proposed, to adapt to the geometry of the surface but still with a large number of voxels, even in regions where the surface is very regular. Moreover, the treatment of singularities remains a (open) problem.

Another family of methods called sample methods have also been used. One type uses moving particles on the surface, with repulsion forces which make it possible to spread the particles over the surface [43]. Another type starts from an initial set of sample points on the surface and refine it by inserting new points of the surface, in order to improve the approximation level. Techniques based on Delaunay triangulation of these points have been used for instance for this purpose $[7,11]$.

In the presence of singularities, these methods are not producing correct output and refining the precision parameter of these algorithms increases the number of output points, without solving these singularity problems.

In a completely different direction, methods inspired by Cylindrical Algebraic Decomposition [9] have been proposed to analyse the topology of algebraic curves or surfaces, even in singular cases. The approach has been applied successfully to curves in 2D, 3D, 4D [23, 27, 22,21,3] and to surfaces [20,8,34]. They use projection techniques based on a conceptual sweeping line/plane perpendicular to some axis, and detect the critical topological events, such as tangents to the sweeping planes and singularities. They involve the exact computation of critical points and genericity condition tests or adjacency tests. The final output of these methods is a topological complex of points, segments, triangles isotopic to the curve or the surface.

They assume exact input equations and rely of the computation of subresultant sequences or calculus with algebraic numbers. This can be a bottleneck in many examples with large degree and large coefficients. Moreover, they are delicate to apply with approximate computation.

In order to combine approximation properties with certification and adaptivity, we consider subdivision methods, which proceed from a large input domain and subdivide it if a regularity criterion is not satisfied. This regularity criterion 
is designed so that the topology of the curve or the surface lying in the domain can be determined easily. Unfortunately, this type of approach has usually difficulties when singular points exists in the domain, which make the regularity test failing and the subdivision process going one until some threshold $\epsilon$ on the size of the boxes is reached. The obstacle comes from the fact that near a singularity, what ever scale of approximation you choose, the shape or topology of the algebraic objects remains similar. In this paper, we will focus on a regularity criterion which allows to deduce the topology of the variety in a domain, from its intersection with the boundary of the domain. We exploit the local conic structure near points on an algebraic curve or surface, to device algorithms which for a small enough threshold $\epsilon$ compute the correct topology, even in the presence of singular points.

These subdivision methods have been already used for solving several equations $[38,15]$. We recall the recent improvements proposed in [33], which rely on a polynomial solver as the basic ingredient of algorithms for curves and surfaces. Extension of this approach to higher dimensional objects have also been considered $[36,27,25,37]$. We will recall the subdivision method described in [28] for curves in $3 \mathrm{D}$. It is based on a criterion, which allows us to detect easily when the topology of the curve in a box is uniquely determined from its intersection with the boundary of the box. The treatment of smooth surfaces by subdivision methods as been described in [2]. In this paper, we extend this approach by encompassing the singular case. The approach relies on the computation of the topology of a special curve on the surface, called the polar variety. It is used to detect points at which the surface has a conic structure, meaning we can tell what the topology is by uniquely looking at its intersection with the boundary of a box around the point.

\section{Definitions}

Before going into details, here are the notations and definitions we use hereafter:

- For subset domain $S \subset \mathbb{R}^{n}$, we denote by $S^{\circ}$ its interior, by $\bar{S}$ its closure, and by $\partial S$ its boundary.

- We call any closed set $D$ such that $\overline{D^{\circ}}=D$, a domain.

- We call any connected smooth curve $\mathcal{C}$ such that $\mathcal{C} \cap \partial D \neq \emptyset$ and $\mathcal{C} \cap D^{\circ} \neq \emptyset$, a branch (relative to a domain $D$ ), .

- We call any connected submanifold (possibly with boundary) included in the surface (resp. curve), with same the dimension as the surface (resp. curve), a patch of a surface (resp. curve).

- We call a point where the tangent space to the surface (resp. curve) contains the direction $x$ (resp. $y, z$ ), a $x$-critical point (resp. $y, z$-critical point) of a surface (resp. curve).

- For any point $p \in \mathbb{R}^{n}$ and $r>0$, the hypersphere (resp. disk) centered at $p$ of radius $r$ is denoted by $S(p, r)=\left\{q \in \mathbb{R}^{n} ;\|q-p\|=r\right\}$ (resp. $\left.D(p, r)=\left\{q \in \mathbb{R}^{n} ;\|q-p\| \leq r\right\}\right)$. 
- By expressions such as "topology computation" or "determine the topology" we mean that we generate an embedded triangulation whose vertices are on the original surface (resp. curve) and which is homeomorphic to that surface (resp. curve). Our construction actually leads to an embedded triangulation that is isotopic (meaning there is a continuous injective deformation of one onto the other) to the original variety, but this would require some more careful examination of our construction.

- For a box $B=\left[a_{0}, b_{0}\right] \times\left[a_{1}, b_{1}\right] \times\left[a_{2}, b_{2}\right] \subset \mathbb{R}^{3}$, its $x$-faces (resp. $y$-face, $z$-face) are its faces normal to the direction $x$ (resp. $y, z)$.

The size of $B$, denoted by $|B|$, is $|B|=\max \left\{\left|b_{i}-a_{i}\right| ; i=1, \ldots, n\right\}$.

\section{Polynomials equations}

This section recalls the theoretical background of Bernstein polynomial representation and how it is related to the problem we want to solve.

\subsection{Bernstein Basis Representation}

Given an arbitrary univariate polynomial function $f(x) \in \mathbb{K}$, we can convert it to a representation of degree $d$ in Bernstein basis, which is defined by:

$$
\begin{aligned}
f(x) & =\sum_{i} b_{i} B_{i}^{d}(x), \text { and } \\
B_{i}^{d}(x) & =\left(\begin{array}{c}
d \\
i
\end{array}\right) x^{i}(1-x)^{d-i}
\end{aligned}
$$

where $b_{i}$ is usually referred as controlling coefficients. Such conversion is done through a basis conversion [17]. The above formula can be generalized to an arbitrary interval $[a, b]$ by a variable substitution $x^{\prime}=(b-a) x+a$. We denote by $B_{d}^{i}(x ; a, b)\left(\begin{array}{l}d \\ i\end{array}\right)(x-a)^{i}(b-x)^{d-i}(b-a)^{-d}$ the corresponding Bernstein basis on $[a, b]$. There are several useful properties regarding Bernstein basis given as follows:

- Convex-Hull Properties: Since $\sum_{i} B_{d}^{i}(x ; a, b) \equiv 1$ and $\forall x \in[a, b], B_{d}^{i}(x ; a, b) \geq$ 0 where $i=0, \ldots, d$, the graph of $f(x)=0$, which is given by $(x, f(x))$, should always lie within the convex-hull defined by the control coefficients $\left(\frac{i}{d}, b_{i}\right)$ $[17]$.

- Subdivision (de Casteljau): Given $t_{0} \in[0,1], f(x)$ can be represented by:

$$
\begin{aligned}
f(x) & =\sum_{i=0}^{d} b_{0}^{(i)} B_{d}^{i}(x ; a, c)=\sum_{i=0}^{d} b_{i}^{(d-i)} B_{d}^{i}(x ; c, b), \text { where } \\
b_{i}^{(k)} & =\left(1-t_{0}\right) b_{i}^{(k-1)}+t_{0} b_{i+1}^{(k-1)} \text { and } c=\left(1-t_{0}\right) a+t_{0} b .
\end{aligned}
$$


By a direct extension to the multivariate case, any polynomial $f\left(x_{1}, \ldots, x_{n}\right) \in$ $\mathbb{R}\left[x_{1}, \ldots, x_{n}\right]$ of degree $d_{i}$ in the variable $x_{i}$, can be decomposed as:

$$
f\left(x_{1}, \ldots, x_{n}\right)=\sum_{i_{1}=0}^{d_{1}} \cdots \sum_{i_{n}=0}^{d_{n}} b_{i_{1}, \ldots, i_{n}} B_{d_{1}}^{i_{1}}\left(x_{1} ; a_{1}, b_{1}\right) \cdots B_{d_{n}}^{i_{n}}\left(x_{n} ; a_{n}, b_{n}\right) .
$$

where $\left(B_{d_{1}}^{i_{1}}\left(x_{1} ; a_{1}, b_{1}\right) \cdots B_{d_{n}}^{i_{n}}\left(x_{n} ; a_{n}, b_{n}\right)\right)_{0 \leq i_{1} \leq d_{1}, \ldots, 0 \leq i_{n} \leq d_{n}}$ is the tensor product Bernstein basis on the domain $B:=\left[a_{1}, b_{1}\right] \times \cdots \times\left[a_{n}, b_{n}\right] \subset \mathbb{R}^{n}$ and $\mathbf{b}=$ $\left(b_{i_{1}, \ldots, i_{n}}\right)_{0 \leq i_{1} \leq d_{1}, \ldots, 0 \leq i_{n} \leq d_{n}}$ are the control coefficients of $f$ on $B$. The polynomial $f$ is represented in this basis by the $n^{\text {th }}$ order tensor of control coefficients $\mathbf{b}=$ $\left(b_{i_{1}, \ldots, i_{n}}\right)_{0 \leq i \leq d_{1}, 0 \leq j \leq d_{2}, 0 \leq k \leq d_{3}}$.

De Casteljau algorithm also applies in each of the direction $x_{i}, i=1, \ldots, n$ so that we can split this representation in these directions. We use the following properties to isolate the roots:

This representation provides a simple way to tell the sign of a function in a domain $B$

Lemma 2.1. If all the coefficients $b_{i_{1}, \ldots, i_{n}}$ of $f$ in Bernstein basis of $B:=$ $\left[a_{1}, b_{1}\right] \times \cdots \times\left[a_{n}, b_{n}\right] \subset \mathbb{R}^{n}$ have the same sign $\epsilon \in\{-1,1\}$, then $\epsilon f(\mathbf{x})>0$ for $\mathbf{x} \in B$.

Proof. As the Bernstein basis elements of the domain $B$ are positive on $B$ and their sum is 1 , for $\mathbf{x} \in B, f(\mathbf{x})$ is a barycentric combination of the coefficients $b_{i_{1}, \ldots, i_{n}}$, of $\operatorname{sign} \epsilon$. This $f(\mathbf{x})$ is of $\operatorname{sign} \epsilon$.

A consequence is the following interesting property:

Lemma 2.2. Let $f$ and $g$ by polynomials of degree $d_{i}$ in $x_{i}(i=1, \ldots, n)$ and let $b_{i_{1}, \ldots, i_{n}}$ and $c_{i_{1}, \ldots, i_{n}}$ be their coefficients in the Bernstein basis of $B:=\left[a_{1}, b_{1}\right] \times$ $\cdots \times\left[a_{n}, b_{n}\right]$. If $b_{i_{1}, \ldots, i_{n}} \leq c_{i_{1}, \ldots, i_{n}}$ for $0 \leq i_{j} \leq d_{j}, j=1, \ldots, n$ then $f(\mathbf{x}) \leq g(\mathbf{x})$ for $\mathbf{x} \in B$.

It will be used in algorithm for computing the topology of implicit curves and surfaces as follows. When the input coefficients of a polynomial $f$ are large rational numbers, instead of working with this expensive arithmetic, we will first compute its coefficients in the Bernstein basis of the given domain $B$, then normalize them and finally round them up and down to machine precision arithmetic (ie. double). This produces two enveloping functions $f, \bar{f}$ with the property:

$$
\underline{f}(\mathbf{x}) \leq f(\mathbf{x}) \leq \bar{f}(\mathbf{x}), \forall \mathbf{x} \in B \text {. }
$$

These two enveloping polynomials can be used to test sign conditions and regularity criteria, providing certificated results in many situations.

\subsection{Univariate Subdivision Solver}

Another interesting property of this representation related to Descartes rule of signs is that there is a simple and yet efficient test for the existence of real 
roots in a given interval. It is based on the number of sign variation $V(\mathbf{b})$ of the sequence $\mathbf{b}=\left[b_{1}, \ldots, b_{k}\right]$ that we define recursively as follows:

$$
V\left(\mathbf{b}_{k+1}\right)=V\left(\mathbf{b}_{k}\right)+\left\{\begin{array}{l}
1, \text { if } b_{i} b_{i+1}<0 \\
0, \text { else }
\end{array}\right.
$$

With this definition, we have:

Proposition 2.1. Given a polynomial $f(x)=\sum_{i}^{n} b_{i} B_{i}^{d}(x ; a, b)$, the number $N$ of real roots of $f$ on $] a, b\left[\right.$ is less than or equal to $V(\boldsymbol{b})$, where $\boldsymbol{b}=\left(b_{i}\right), i=1, \ldots, n$ and $N \equiv V(\boldsymbol{b}) \bmod 2$.

With this proposition,

- if $V(\mathbf{b})=0$, the number of real roots of $f$ in $[a, b]$ is 0 ;

- if $V(\mathbf{b})=1$, the number of real roots of $f$ in $[a, b]$ is 1 .

This yields the following simple and efficient algorithm [16]:

Algorithm 2.1

INPUT: A precision $\epsilon$ and a polynomial $f$ represented in the Bernstein basis of an interval $[b, a]: f=(\boldsymbol{b},[a, b])$.

- Compute the number of sign changes $V(\boldsymbol{b})$.

- If $V(\boldsymbol{b})>1$ and $|b-a|>\epsilon$, subdivide the representation into two subrepresentations $\boldsymbol{b}^{-}, \boldsymbol{b}^{+}$, corresponding to the two halves of the input interval and apply recursively the algorithm to them.

- If $V(\boldsymbol{b})>1$ and $|b-a|<\epsilon$, output the $\epsilon / 2$-root $(a+b) / 2$ with multiplicity $V(\boldsymbol{b})$.

- If $V(\boldsymbol{b})=0$, remove the interval $[a, b]$.

- If $V(\boldsymbol{b})=1$, the interval contains one root, that can be isolated with precision $\epsilon$.

OUTPUT: list of subintervals of $[a, b]$ containing exactly one real root of $f$ or of $\epsilon$-roots with their multiplicities.

In the presence of a multiple root, the number of sign changes of a representation containing a multiple root is bigger than 2 , and the algorithm splits the box until its size is smaller than $\epsilon$.

In order to analyze the behavior of the algorithm, a partial inverse of Descartes' rule and lower bounds on the distance between roots of a polynomial have been used. It is proved that the complexity of isolating the roots of a polynomial of degree $d$, with integer coefficients of bit size $\leq \tau$ is bounded by $\mathcal{O}\left(d^{4} \tau^{2}\right)$ up to some polylog factors. See $[13,16]$ for more details.

Notice that this localization algorithm extends naturally to B-splines, which are piecewise polynomial functions [17].

The approach can also be extended to polynomials with interval coefficients, by counting 1 sign variation for a sign sub-sequence $+, ?,-$ or $-, ?,+; 2$ sign variations for a sign sub-sequence $+, ?,+$ or $-, ?,-; 1$ sign variation for a sign sub-sequence ?,?, where ? is the sign of an interval containing 0. Again in this case, if a family $\bar{f}$ of polynomials is represented by the sequence of intervals $\overline{\mathbf{b}}=\left[\bar{b}_{0}, \ldots, \bar{b}_{d}\right]$ in the Bernstein basis of the interval $[a, b]$ 
- if $V(\overline{\mathbf{b}})=1$, all the polynomials of the family $\bar{f}$ have one root in $[a, b]$,

- if $V(\overline{\mathbf{b}})=0$, all the polynomials of the family $\bar{f}$ have no roots in $[a, b]$.

The same subdivision algorithm can be applied to polynomials with interval coefficients, using interval arithmetic. This yields either intervals of size smaller than $\epsilon$, which might contain the roots of $f=0$ in $[a, b]$ or isolating intervals for all the polynomials of the family defined by the interval coefficients.

\subsection{Multivariate Bernstein Subdivision solver}

We consider here the problem of computing the solutions of a polynomial system

$$
\left\{\begin{array}{l}
f_{1}\left(x_{1}, \ldots, x_{n}\right)=0 \\
\vdots \\
f_{s}\left(x_{1}, \ldots, x_{n}\right)=0
\end{array}\right.
$$

in a box $B:=\left[a_{1}, b_{1}\right] \times \cdots \times\left[a_{n}, b_{n}\right] \subset \mathbb{R}^{n}$. The method for approximating the real roots of this system, that we describe now uses the representation of multivariate polynomials in Bernstein basis, analysis of sign variations and univariate solvers (section 2.2). The output is a set of small-enough boxes, which contain these roots. This subdivision solver which can be seen as an improvement of the Interval Projected Polyhedron algorithm in [38], it is described in more details in [33].

In the following, we use the Bernstein basis representation of a multivariate polynomial $f$ of the domain $I:=\left[a_{1}, b_{1}\right] \times \cdots \times\left[a_{n}, b_{n}\right] \subset \mathbb{R}^{n}$ :

$$
f\left(x_{1}, \ldots, x_{n}\right)=\sum_{i_{1}=0}^{d_{1}} \cdots \sum_{i_{n}=0}^{d_{n}} b_{i_{1}, \ldots, i_{n}} B_{d_{1}}^{i_{1}}\left(x_{1} ; a_{1}, b_{1}\right) \cdots B_{d_{n}}^{i_{n}} x\left(x_{n} ; a_{n}, b_{n}\right) .
$$

Definition 2.1. For any $f \in \mathbb{R}[\mathbf{x}]$ and $j=1, \ldots, n$, let

$$
\begin{aligned}
& m_{j}\left(f ; x_{j}\right)=\sum_{i_{j}=0}^{d_{j}} \min _{\left\{0 \leq i_{k} \leq d_{k}, k \neq j\right\}} b_{i_{1}, \ldots, i_{n}} B_{d_{j}}^{i_{j}}\left(x_{j} ; a_{j}, b_{j}\right) \\
& M_{j}\left(f ; x_{j}\right)=\sum_{i_{j}=0}^{d_{j}} \max _{\left\{0 \leq i_{k} \leq d_{k}, k \neq j\right\}} b_{i_{1}, \ldots, i_{n}} x B_{d_{j}}^{i_{j}}\left(x_{j} ; a_{j}, b_{j}\right) .
\end{aligned}
$$

Theorem 2.1 (Projection Lemma). For any $\mathbf{u}=\left(u_{1}, \ldots, u_{n}\right) \in I$, and any $j=1, \ldots, n$, we have

$$
m\left(f ; u_{j}\right) \leq f(\mathbf{u}) \leq M\left(f ; u_{j}\right) .
$$

As a direct consequence, we obtain the following corollary:

Corollary 2.1. For any root $\mathbf{u}=\left(u_{1}, \ldots, u_{n}\right)$ of the equation $f(\mathbf{x})=0$ in the domain $I$, we have $\underline{\mu}_{j} \leq u_{j} \leq \bar{\mu}_{j}$ where 
- $\underline{\mu}_{j}\left(\right.$ resp. $\left.\bar{\mu}_{j}\right)$ is either a root of $m_{j}\left(f ; x_{j}\right)=0$ or $M_{j}\left(f ; x_{j}\right)=0$ in $\left[a_{j}, b_{j}\right]$ or

$a_{j}$ (resp. $\left.b_{j}\right)$ if $m_{j}\left(f ; x_{j}\right)=0$ (resp. $\left.M_{j}\left(f ; x_{j}\right)=0\right)$ has no root on $\left[a_{j}, b_{j}\right]$,

- $m_{j}(f ; u) \leq 0 \leq M_{j}(f ; u)$ on $\left[\underline{\mu}_{j}, \bar{\mu}_{j}\right]$.

The solver implementation contains the following main steps. It consists in

1. applying a preconditioning step to the equations;

2. reducing the domain;

3. if the reduction ratio is too small, to split the domain

until the size of the domain is smaller than a given epsilon.

The following important ingredients of the algorithm parameterize its implementation:

Preconditioner. It is a transformation of the initial system into a system, which has a better numerical behavior. Solving the system $\mathbf{f}=0$ is equivalent to solving the system $M \mathbf{f}=0$, where $M$ is an $s \times s$ invertible matrix As such a transformation may increase the degree of some equations, with respect to some variables, it has a cost, which might not be negligible in some cases. Moreover, if for each polynomial of the system not all the variables are involved, that is if the system is sparse with respect to the variables, such a preconditioner may transform it into a system which is not sparse anymore. In this case, we would prefer a partial preconditioner on a subsets of the equations sharing a subset of variables. We consider Global transformations, which minimize the distance between the equations, considered as vectors in an affine space of polynomials of a given degree and Local straightening (for $s=n$ ), which transform locally the system $\mathbf{f}$ into a system $J^{-1} \mathbf{f}$, where $J=\left(\partial_{x_{i}} f_{j}\left(\mathbf{u}_{0}\right)\right)_{1 \leq i, j \leq s}$ is the Jacobian matrix of $\mathbf{f}$ at a point $\mathbf{u}$ of the domain $I$, where it is invertible.

It can be proved that the reduction based on the polynomial bounds $m$ and $M$ behaves like Newton iteration near a simple root, that is we have a quadratic convergence, with this transformation.

Reduction strategy, that is the technique used to reduce the initial domain, for searching the roots of the system. It can be based on Convex hull properties as in [38] or on Root localisation, which is a direct improvement of the convex hull reduction and consists in computing the first (resp. last) root of the polynomial $m_{j}\left(f_{k} ; u_{j}\right)$, (resp. $\left.M_{j}\left(f_{k} ; u_{j}\right)\right)$, in the interval $\left[a_{j}, b_{j}\right]$. The current implementation of this reduction steps allows us to consider the convex hull reduction, as one iteration step of this reduction process.

The guarantee that the computed intervals contain the roots of $f$, is obtained by controlling the rounding mode of the operations during the de Casteljau computation.

Subdivision strategy, that is technique used to subdivide the domain, in order to simplify the forthcoming steps, for searching the roots of the system. The approach, that we are using in our implementation is the parameter domain bisection: The domain $b$ is then split in half in a direction $j$ for which $\left|b_{j}-a_{j}\right|$ 
is maximal. But instead of choosing the size of the interval as a criterion for the direction in which we split, we may choose other criterion depending also on the value the functions $m_{i}, M_{j}$ or $f_{j}$ (for instance where $M_{j}-m_{j}$ is maximal).

A bound for the complexity of this method is detailed in [33]. It involves metric quantities related to the system $\mathbf{f}=0$, such as the Lipschitz constant of f in $B$, the entropy of its near-zero level sets, a bound $d$ on the degree of the equations in each variable and the dimension $n$.

\subsection{Example}

Here are some comparisons of the different strategies, describing the number of iterations in the main loop, the number of subdivision of a domain, the number of boxes produced by the method, the time it takes. We compare the method sbd, a pure subdivision approach, rd a method doing first reduction and based on a univariate root-solver using the Descarte's rule. sbds], a subdivision approach using the global preconditioner, rds, a reduction approach using the global preconditioner, rdl, a reduction approach using the jacobian preconditioner (see Fig. 1). The first example is a bivariate system, with equations of degree 12 in each variable, the second example is of bidegree $(8,8)$. For more details on this

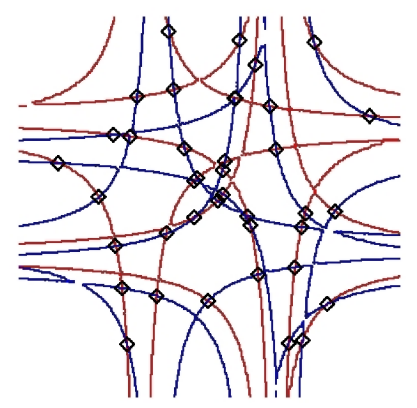

\begin{tabular}{|l|c|c|c|c|}
\hline method & iterations & subdivisions & results & time $(\mathrm{ms})$ \\
\hline sbd & 4826 & 4826 & 220 & 217 \\
rd & 2071 & 1437 & 128 & 114 \\
sbds & 3286 & 3286 & 152 & 180 \\
rds & 1113 & 748 & 88 & 117 \\
rdl & 389 & 116 & 78 & 44 \\
\hline
\end{tabular}

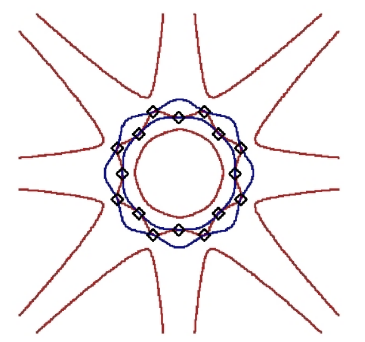

\begin{tabular}{|l|c|c|c|c|}
\hline method & iterations & subdivisions & results & time $(\mathrm{ms})$ \\
\hline sbd & 84887 & 84887 & 28896 & 3820 \\
rd & 82873 & 51100 & 20336 & 4553 \\
sbds & 6076 & 6076 & 364 & 333 \\
rds & 1486 & 920 & 144 & 163 \\
rdl & 1055 & 305 & 60 & 120 \\
\hline
\end{tabular}

Fig. 1. Behavior of the subdivision solver for different preconditioners.

solver, see $[35,33]$. 


\section{Planar curves}

In this section, we consider a curve $\mathcal{C}$ in $\mathbb{R}^{2}$, defined by the equation $f(x, y)=0$ with $f \in \mathbb{Q}[x, y]$ and a domain $B=[a, b] \times[c, d] \subset \mathbb{R}^{2}$.

\subsection{Regularity criterion for planar curves}

We recall that a tangent to the curve $\mathcal{C}$ is a line, which intersects $\mathcal{C}$ with multiplicity $\geq 2$. In particular, any line through a singular point of $\mathcal{C}$ is tangent to $\mathcal{C}$.

Definition 3.1. We say that the curve $\mathcal{C}$ is y-regular (resp. x-regular) in $B$, if $\mathcal{C}$ has no tangent parallel to the $y$-direction (resp. x-direction) in $B$.

Notice that if $\mathcal{C}$ is $x$-regular (or $y$-regular) it is smooth in $B$ since it cannot have singular points in $B$. A curve is regular in $B$, if it is $x$-regular or $y$-regular in $B$.

We are going to show that if $\mathcal{C}$ is $x$-regular in $B$, then its topology can be deduced from its intersection with the boundary $\partial B$.

Definition 3.2. For a point $p \in \mathcal{C} \cap \partial B$, we define its interior tangent $T_{p}^{i}(\mathcal{C})$ as the tangent of $\mathcal{C}$ at $p$, pointing inside $B$.

If the curve is not tangent to $\partial B$ at $p \in \partial B$ and $p$ is not a corner point of $B$, this direction is defined by $\epsilon_{p} T_{p}(\mathcal{C})$ with $\epsilon_{p}=\operatorname{sign}\left(T_{p}(\mathcal{C}) \cdot \nu_{p}\right)$, where $\nu_{p}$ is the unit normal interior vector to $\partial B$ at $p$.

If the curve is tangent to $\partial B$ at $p$, we say that the point is of multiplicity 2. In this case, we consider the half branches of $\mathcal{C}$ at $p$, and associate to each of them the unit tangent vector to this branch, if the branch is inside $B$ near $p$. In the following, if two opposite unit vectors are attached to a point $p$, we will duplicate this point, so that a point is attached to a unique interior tangent.

If $p$ is at the corner of $B$, we extend this definition, as follows: We consider the cone of interior normal vectors $\nu_{p}$ of $B$ at $p$, and require that $T_{p}^{i}(\mathcal{C}) \cdot \nu_{p} \geq 0$ for all the vectors $\nu_{p}$ in this cone. Thus, this interior tangent might not exist for corner points.

Definition 3.3. For a point $p \in \mathcal{C} \cap \partial B$ with interior tangent $T_{p}^{i}(\mathcal{C})$, we define its $x$-index (resp. $y$-index) as $\operatorname{sign}\left(T_{p}^{i}(\mathcal{C}) \cdot e_{1}\right)$ where $e_{1}$ is the unit vector $(1,0) \in \mathbb{R}^{2}$ (resp. $(0,1) \in \mathbb{R}^{2}$. If the interior tangent of $\mathcal{C}$ at $p$ does not exist, we define the $x$-index of $p$ as 0 .

For a $y$-regular curve $\mathcal{C}$ (with no vertical tangent) in $B$ and $p \in](a, c),(a, d)[$, we have $x$-index $(p)=1$. If $p \in](b, c),(b, d)[$, its $x$-index is -1 . Moreover, if the curve is not tangent to the horizontal segment on the boundary of $B$, the $x$-index of a point of $\mathcal{C} \cap \partial P$ which is not a corner point of $B$ is not 0 .

Lemma 3.1. If $\mathcal{C}$ is $y$-regular in $B$, then a branch of $\mathcal{C} \cap B$ connects a point $p$ of $x$-index 1 to a point $q$ of $x$-index -1 , such that $x_{p}<x_{q}$. 
Proof. As the curve is $y$-regular, it has no vertical tangent and thus no closed loop in $B$. Consequently, each of the interior connected components of $\mathcal{C} \cap B$ intersects $\partial B$ in two distinct points $p, q \in \mathcal{C} \cap \partial B$ (with $x_{p} \leq x_{q}$ ).

Assume that the $x$-index of $p, q$ are the same. Suppose that this index is 1 . Then for an analytic parameterisation $s \in[0,1] \mapsto(x(s), y(s))$ of the branch $[p, q]$ with $(x(0), y(0))=p,(x(1), y(1))=q$, we have $\partial_{s} x(0)>0, \partial_{s} x(1)<0$. This implies that for a value $0<s_{0}<1, x\left(s_{0}\right)>x(1)=x_{q} \geq x(0)=x_{p}$ and that there exists $\left.s_{0}^{\prime} \in\right] 0,1\left[\right.$ such that $x\left(s_{0}^{\prime}\right)=x(1)$. We deduce that $\partial_{s} x(s)$ vanishes in $[0,1]$ and that the branch $[p, q]$ of $\mathcal{C}$ has a vertical tangent, which is excluded by hypothesis. If the index of $p$ and $q$ is -1 , we exchange the role of $p$ and $q$ and obtain the same contradiction. As $\partial_{s} x(s)>0$ for $s \in[0,1]$, we have $x_{p}<x_{q}$, which proves the lemma.

Lemma 3.2. Suppose that $\mathcal{C}$ is y-regular in $B$ and let $p, q$ be two consecutive points of $\mathcal{C} \cap \partial B$ with

$$
\begin{aligned}
& -x \text {-index }(p)=1, x \text {-index }(q)=-1, \\
& -x_{p}<x_{q} .
\end{aligned}
$$

Then $p, q$ belong to the same branch of $\mathcal{C} \cap B$.

Proof. Let $p, q$ be two consecutive points of $\mathcal{C} \cap \partial B$, with $x$-index $(p)=1, x$ index $(q)=-1$ and $x_{p}<x_{q}$. Suppose that $p, q$ belong to two branches $\left(p, p^{\prime}\right)$, $\left(q, q^{\prime}\right)$ of $\mathcal{C}$ in $B$. As the curve $\mathcal{C}$ is smooth in $B$, the two branches do not intersect, and the points $q, q^{\prime}$ are on the same component of $\partial B-\left\{p, p^{\prime}\right\}$. As $x$-index $(q)=-1$, by the previous lemma $x$-index $\left(q^{\prime \prime}\right)=1$ and $x\left(q^{\prime}\right)<x(q)$. This implies that $q^{\prime}$ is a point in-between $p$ and $q$ on $\partial B$, on one component of $\partial B-\{p, q\}$. A similar argument shows that $p^{\prime}$ is a point between $p$ and $q$ on $\partial B$ on the other component of $\partial B-\{p, q\}$. This contradicts the fact that $p$ and $q$ are consecutive points of $\partial B$.

Proposition 3.1. If $\mathcal{C}$ is regular in $B$, its topology in $B$ is uniquely determined by its intersection $\mathcal{C} \cap \partial B$ with the boundary of $B$.

Proof. Exchanging the role of $x$ and $y$ if necessary, we can assume that $\mathcal{C}$ is $y$-regular. We prove the proposition by induction on the number $N(\mathcal{C})$ of points on $\mathcal{C} \cap \partial_{B}$, with non-zero $x$-index. We denote this set of points by $\mathcal{L}$.

Since the curve has no vertical tangent in $B$ and has no closed loop, each of the connected components of $\mathcal{C} \cap B$ intersects $\partial B$ in two distinct points of $x$-index $\neq 0$. Thus if $N(\mathcal{C})=0$, then there is no branch of $\mathcal{C}$ in $B$.

Assume now that $N(\mathcal{C})>0$, and let us show that it is possible to find two consecutive points $p, q$ of $\mathcal{L}$ with $x$-index $(p)=1, x$-index $(q)=-1, x_{p}<x_{q}$. As the curve $\mathcal{C}$ is smooth in $B$, its $k$ branches are not intersecting each other and they split $B$ into $k+1$ connected components, which intersect the boundary $\partial B$. Consider a branch $[p, q]$ which separates $k$ of these components from the last one. Then, there is no other points of $\mathcal{C} \cap \partial B$ in-between $p$ and $q$. By lemma 3.2, we have $x$-index $(p)=1, x$-index $(q)=-1, x_{p}<x_{q}$. 


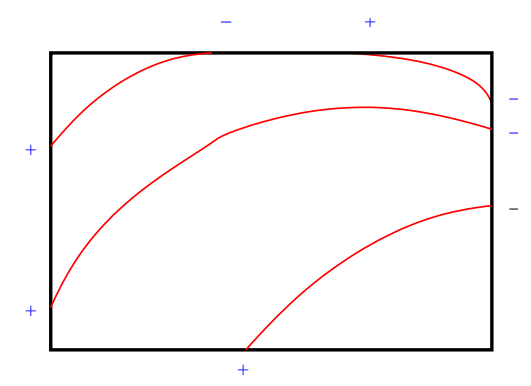

Fig. 2. Illustration of Proposition 3.1.

Removing this branch from $\mathcal{C}$, we obtain a new curve $\mathcal{C}^{\prime}$ which is still $y$ regular and such that $N\left(\mathcal{C}^{\prime}\right)<N(\mathcal{C})$. We conclude by induction hypothesis, that the topology of $\mathcal{C}^{\prime}$ and thus of $\mathcal{C}$ is uniquely determined.

If $\mathcal{C}$ is $y$-regular, by exchanging the role of $x$ and $y$, we can deduce the topology in $B$ from the points on $\partial B$. The initial domain can be subdivided in such a way, that each box contains either a unique singular point and is small enough or is regular. For the small boxes $B$ containing a unique singular point, we are going to connect the center of $B$ with the points of $\mathcal{C} \cap \partial B$. For the regular boxes, we apply the following algorithm:

\section{Algorithm 3.1 (Connection for a regular curve)}

INPUT: an algebraic curve $\mathcal{C}$ and a domain $B=[a, b] \times[c, d] \subset \mathbb{R}^{2}$ such that $\mathcal{C}$ has no vertical (resp. horizontal) tangent in $B$.

- Isolate the points $\mathcal{C} \cap \partial B$ and compute their $x$-index (or $y$-index);

- Order the points of $\mathcal{C} \cap \partial B$ with non-zero $x$-indices (resp. $y$-indices); clockwise and store them in the circular list $\mathcal{L}$.

- While $\mathcal{L}$ is not empty,

- find two consecutive points $p, q$ in $\mathcal{L}$ with $x$-index $(p)=1, x$-index $(q)=$ $-1, x_{p}<x_{q}$ (resp. $y$-index $(p)=1, y$-index $\left.(q)=-1, y_{p}<y_{q}\right)$;

- add the arc $[p, q]$ to the set $\mathcal{B}$ of branches and remove $p, q$ from $\mathcal{L}$.

Output: the set $\mathcal{B}$ of branches of $\mathcal{C}$ in $B$.

\subsection{Tests of regularity}

Here are simple tests to check the regularity of a curve in a domain, which extends in some way the criterion in [19].

Proposition 3.2. If $\partial_{y} f(x, y) \neq 0$ (resp. $\partial_{x} f(x, y) \neq 0$ ) in a domain $B=$ $\left[a_{0}, b_{0}\right] \times\left[a_{1}, b_{1}\right] \subset \mathbb{R}^{2}$, the curve $\mathcal{C}$ is regular on $B$.

Proof. If $\partial_{y} f \neq 0$ in $B$, the curve $\mathcal{C}$ cannot have vertical tangent and is thus $y$-regular.

The representation of polynomials in Bernstein basis can be used to verify easily this condition. 
Proposition 3.3. If the coefficients of $\partial_{y} f(x, y) \neq 0$ (resp. $\partial_{x} f(x, y) \neq 0$ ) in the Bernstein basis of the domain $B=[a, b] \times[c, d] \subset \mathbb{R}^{2}$ have the same sign $\in\{-1,1\}$, then the curve $\mathcal{C}$ is regular on $B$.

Proof. If the Bernstein coefficients of $\partial_{y} f\left(\right.$ resp. $\left.\partial_{x} f\right)$ are all $>0($ resp. $<0)$, so is $\partial_{y} f$ (resp. $\left.\partial_{x} f\right)$ in $B$ (by the convex hull property), which implies that the curve $f(x, y)=0$ is regular in $B$.

Suppose that $\partial_{y} f(x, y) \neq 0$ in $B$. Then two branches of $\mathcal{C}$ in $B$ cannot have points with the same $x$-coordinate (otherwise $\partial_{y} f$ would vanish in-between these two points). Consequently, the branches do not overlap by projection on the $x$-axis and the connection algorithm can be simplified as follows:

- Compute the points of $\mathcal{C} \cap \partial B$, repeating a point if its multiplicity is even.

- Sort them by lexicographic order so that $x>y: \mathcal{L}:=\left\{p_{1}, p_{2}, \ldots\right\}$

- Connect them by pair $\left[p_{1}, p_{2}\right],\left[p_{3}, p_{4}\right], \ldots$ of consecutive points in $\mathcal{L}$.

\subsection{Algorithm of subdivision}

This yields the following new subdivision algorithm for a planar implicit curve:

\section{Algorithm 3.2 (Topology of a planar implicit curve)}

INPUT: $A$ domain $B_{0} \subset \mathbb{R}^{2}$, a curve $\mathcal{C}$ defined by the squarefree polynomial equation $f(x, y)=0$ with $f \in \mathbb{Q}[x, y]$ and $\epsilon>0$.

- Compute the $x$ and $y$ critical points of $f(x, y)=0$.

$-\mathcal{L}=\left\{B_{0}\right\}$

- While $\mathcal{L}$ is not empty,

- Choose and remove a domain $B$ of $\mathcal{L}$;

- Test if there is a unique critical point, which is not singular in B;

- If it is the case, compute the topology of $\mathcal{C}$ in B (Algorithm 3.1),

- else if $|B|>\epsilon$, subdivide the box into subdomains and add them to $\mathcal{L}$,

- otherwise connect the center of the box to the points of $\mathcal{C} \cap \partial B$.

OUTPUT: a set of points and a set of (smooth) arcs connecting these points.

An interesting advantage of this algorithm compared to sweeping algorithms (such as [22]) is that it avoids a projection on a line, which requires (sub)resultant computations and the manipulation of algebraic numbers.

A variant of this algorithm consists in filtering the regularity test by sign tests (Proposition 3.3) on the Bernstein representation of $f$ in $B$ and to compute the $x$ and $y$ critical points only in domains $B$ where the Bernstein coefficients of $f$ and $\partial_{x} f, \partial_{y} f$ have sign changes.

Proposition 3.4. For $\epsilon>0$ small enough, the algorithm 3.2 computes a graph of points which is isotopic to the curve $\mathcal{C} \cap B$. 
Proof. By Proposition 3.2, if the box $B$ contains no singular points and at most one (smooth) $x$ or $y$ critical point, the topology of the curve is uniquely determined by its intersection with $\partial B$. In these domains, the algorithm produce the correct topological graph.

Suppose that the algorithm treats a domain $B$ which contains a singular point $p$. Then its size is smaller than $\epsilon$. We are going to show that for $\epsilon$ small enough, the topology of the curve in $B$ is given by the graph connecting a point inside to the points of $\mathcal{C} \cap \partial B$. Let us first choose $\epsilon^{\prime}$ smaller than half the minimal distance between two distinct $x$ or $y$ critical points of $\mathcal{C}$ so that $B$ contains only one singular point $p$ of $\mathcal{C}$.

Using Puiseux expansion at the singular point $p[1,41]$, we can construct a disk $D\left(p, \epsilon^{\prime}\right)$ of radius $\epsilon^{\prime}$, centered at $p$, in which the curve $\mathcal{C}$ is the union of real branches described by the image of analytic functions $\sigma_{i}, i=1, \ldots, r$ over the interval $[0,1]$, with $\sigma_{i}(0)=p$ and $\sigma_{i}(1) \in \partial D\left(p, \epsilon^{\prime}\right)$. Since in this disk, the only critical point of $\mathcal{C}$ is $p$, these branches have no tangents parallel to the $x$ or $y$-axis on the interval $] 0,1[$.

Let us choose a box $B$ of size $<\epsilon=\frac{1}{\sqrt{2}} \epsilon^{\prime}$ with $p \in B^{\circ}$. Then $B \subset D\left(p, \epsilon^{\prime}\right)$. As $\sigma_{i}(0)=p \in B^{\circ}$ and $\sigma_{i}(1) \in \partial D\left(p, \epsilon^{\prime}\right)$, each branch intersects $\partial B$. Suppose now that a given branch intersects $\partial B$ in 2 or more points $\sigma_{i}\left(t_{0}\right) \sigma_{i}\left(t_{1}\right)$ with $0 \leq t_{0}<t_{1} \leq 1$. Then there is a value $t_{0} \leq t \leq t_{1}$ such that the tangent at $t$ is parallel to one of the $x$ or $y$ directions, which is excluded. This implies that the number of real branches at $p$ and the number of points of $\mathcal{C} \cap \partial B$ coincides for a $B$ of size $<\epsilon$, with $p \in B^{\circ}$.

Consequently, for $\epsilon$ small enough, the algorithm compute the correct topological graph.

Remark 3.1. The number of branches at a singular point can be computed from information on the boundary of the domain by using the topological degree [29]. Thus, it is possible to control effectively when the number of points of $\mathcal{C} \cap \partial B$ is the number of branches at the singular point $p$ and in this way, to certify the result of this algorithm. But describing these techniques would lead us too far outside the scope of this paper.

Examples are shown in Figures 3 and 4.

\section{Space curves}

In this section, we consider a curve $\mathcal{C}$ of $\mathbb{R}^{3}$. We suppose that $I(\mathcal{C})=\left(f_{1}, f_{2}, \ldots, f_{k}\right)$ and for two polynomials $f(x, y, z), g(x, y, z) \in I(\mathcal{C})$, we define $\mathbf{t}=\nabla(f) \wedge \nabla(g)$ We are interested in the topology of $\mathcal{C}$ in a box $B=\left[a_{0}, b_{0}\right] \times\left[a_{1}, b_{1}\right] \times\left[a_{2}, b_{2}\right]$. Similar to the $2 \mathrm{D}$ case, we can represent $f, g$ and each component of $\mathbf{t}$ in the Bernstein basis for the domain $B$. As we will see, the sign changes of the resulting Bernstein coefficients will make it possible to test the regularity of the curve with minimal effort. 

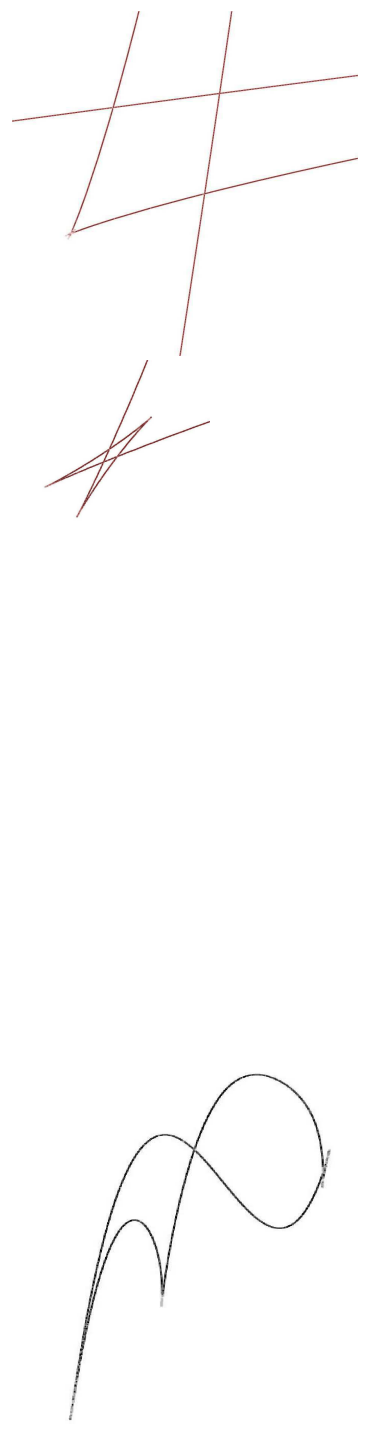

This curve is the discriminant curve of a bivariate system with few monomials used in [12] to give a counter-example to Kushnirenko's conjecture. It is of degree 47 in $x$ and $y$, and the maximal bit size of its coefficient is of order 300. It takes less that 10 s to compute the topological graph, by rounding up and down to the nearest double machine precision numbers, applying the subdivision techniques on the enveloping polynomials. We observe a very tiny domain, which at first sight looks like a cusp point, but which contains in reality, 3 cusps points and 3 crossing points. The central region near these cusp points is the region where counter-examples have been found.

Fig. 3. A discriminant curve.

This curve is the projection onto the $(x, y)$ plane of the curve of points with tangent parallel to the $z$-direction for a surface of degree 4 . It is defined by the equation of degree 12 , and has 4 real cusps and 2 real crossing points. The size of the coefficients is small and the topological graph is computed in less than 1 second. It defines 4 connected regions in the plane, one of these being very small and difficult to see on the picture.

Fig. 4. The apparent contour of a quartic surface. 


\subsection{Regularity criterion for curves in 3D}

In this section, we recall some criteria for the regularity of space curves. More details can be found in [28]. These criteria are based on the following property:

Proposition 4.1. If $\mathcal{C}$ is smooth in $B$ and if for all $x_{0} \in \mathbb{R}$, the plane $x=x_{0}$ has at most one intersection point with the curve $\mathcal{C}$ in $B$, then the topology of $\mathcal{C}$ is uniquely determined from the points $\mathcal{C} \cap \partial B$.

Proof. Consider the projection $\pi_{z}(\mathcal{C})$ of the curve $\mathcal{C}$ in $B$ along the $z$ direction. Then the components of $\mathcal{C}$ in $B$ projects bijectively on the $(y, z)$ plane. Otherwise, there exist two points $p_{0}$ and $p_{1}$ lying on $\mathcal{C}$ such that $\pi_{z}\left(p_{0}\right)=\pi_{z}\left(p_{1}\right)=\left(x_{0}, y_{0}\right)$, then $p_{0}$ and $p_{1}$ belong to $x=x_{0}$ which are functions of the form $y=\Phi(x)$. Otherwise, there exist two points on $\pi_{z}(\mathcal{C})$ and (and on $\mathcal{C} \cap B$ ) with the same $x$-coordinate. Consequently, for $x \in\left[a_{0}, b_{0}\right]$ there is at most one branch of $\pi_{z}(\mathcal{C})$ in $B$ above $x$, and the connected components of $\mathcal{C} \cap B^{\circ}$ project bijectively onto non-overlapping open intervals of $\left[a_{0}, b_{0}\right]$ as $\pi_{z}(\mathcal{C})$ does. We conclude as in the $2 \mathrm{D}$ case (proposition 3.3), by sorting the points of $\mathcal{C} \cap \partial B$ according to their $x$-coordinates, and by grouping them in consecutive pairs corresponding to the start and end points of the branches of $\mathcal{C} \cap B$.

Here is an explicit way to check the conditions of Proposition 4.1:

Proposition 4.2. The $3 D$ spatial curve $\mathcal{C}$ defined by $f=0$ and $g=0$ is regular on $B$, if

- $\boldsymbol{t}_{x}(\boldsymbol{x}) \neq 0$ on $B$, and

$-\partial_{y} h \neq 0$ on $z$-faces, and $\partial_{z} h \neq 0$ and it has the same sign on both $y$-faces of $B$, for $h=f$ or $h=g$.

Proof. Let us fix $x_{0} \in\left[a_{0}, b_{0}\right]$ where $B=\left[a_{0}, b_{0}\right] \times\left[a_{1}, b_{1}\right] \times\left[a_{2}, b_{2}\right]$, let $U=$ $\left\{x_{0}\right\} \times\left[a_{1}, b_{1}\right] \times\left[a_{2}, b_{2}\right]$ and let $\Phi_{x_{0}}:\left(x_{0}, y, z\right) \in U \mapsto\left(f\left(x_{0}, y, z\right), g\left(x_{0}, y, z\right)\right)$. We are going to prove that under our hypothesis, $\Phi_{x_{0}}$ is injective. The Jacobian $\mathbf{t}_{x}\left(x_{0}, y, z\right)$ of $\Phi_{x_{0}}$ does not vanish on $U$, so that $\Phi_{x_{0}}$ is locally injective. We consider the level-set $f(\mathbf{x})=f_{0}$ for some $f_{0} \in f(U)$. It cannot contain a closed loop in $U$, otherwise we would have $\left(\partial_{y} f, \partial_{z} f\right)=0$ (and thus $\mathbf{t}_{x}=0$ ) in $U \subset B$. We deduce that each connected component of $f(\mathbf{x})=f_{0}$ in $U$ intersects $\partial U$ in two points.

Now suppose that $\Phi_{x_{0}}$ is not injective on $U$, so that we have two points $p_{1}, p_{2} \in U$ such that $\Phi_{x_{0}}\left(p_{1}\right)=\Phi_{x_{0}}\left(p_{2}\right)$.

If $p_{1}$ and $p_{2}$ are on the same connected component of the level set $f(\mathbf{x})=f_{0}$ (where $\left.f_{0}=f\left(p_{1}\right)=f\left(p_{2}\right)\right)$ in $U$, then $g$ reaches the same value at $p_{1}$ and $p_{2}$ on this level set, so that by Role's theorem, there exists a point $p \in U$ between $p_{1}$ and $p_{2}$, such that $\operatorname{Jac}\left(\Phi_{x_{0}}\right)(p)=\mathbf{t}_{x}(p)=0$. By hypothesis, this is impossible.

Thus $p_{1}$ and $p_{2}$ belongs to two different connected components of $f(\mathbf{x})=f_{0}$ in $U$. Consequently the value $f_{0}$ is reached at 4 distinct points of $\partial U$, which implies that $f$ has at least 4 extrema on $\partial U$.

Now note that up to a change of variable $z=a_{2}-z$, we can assume that $\partial_{z} f>0$ on both $y=a_{1}, y=b_{1}$ faces. Then if $\partial_{y} f<0$ on $z=a_{2}$, we have 
$f\left(x_{0}, a_{1}, b_{2}\right)>f\left(x_{0}, a_{1}, a_{2}\right)>f\left(x_{0}, b_{1}, a_{2}\right)$ and $\left(a_{2}, a_{3}\right)$ is not a local extremum. Otherwise $\partial_{y} f>0$ and $\left(b_{1}, a_{2}\right)$ is not a local extremum. In both cases, we do not have 4 extrema, which proves that $\sigma_{x_{0}}$ is injective and that the intersection of $\mathcal{C}$ with the plane $x=x_{0}$ in $B$ is at most one point. So by proposition 4.1 , we deduce that $\mathcal{C}$ is regular in $B$.

A similar criterion applies by symmetry, exchanging the roles of the $x, y, z$ coordinates. If one of these criteria applies with $\mathbf{t}_{i}(x) \neq 0$ on $B$ (for $i=x, y, z$ ), we will say that $\mathcal{C}$ is $i$-regular on $B$.

\subsection{Tests of regularity}

From a practical point of view, the test $\mathbf{t}_{i}(x) \neq 0$ or $\partial_{i}(h) \neq 0$ for $i=x, y$ or $z$, $h=f$ or $g$ can be replaced by the stronger condition that their coefficients on the Bernstein basis of $B$ have a constant sign, which is straightforward to check. Similarly, such a property on the faces of $B$ is also direct, since the coefficients of a polynomial on a facet form a subset of the coefficients of this polynomial in the box.

In addition to these tests, we also test whether both surfaces penetrate the cell, since a point on the curve must lie on both surfaces. This test could be done by looking at the sign changes of the Bernstein coefficients of the surfaces with respect to that cell. If no sign change occurs, we can rule out the possibility that the cell contains any portion of the curve $\mathcal{C}$, and thus terminate the subdivision early. In this case, we will also say that the cell is regular.

\subsection{Algorithm of connection}

This regularity criterion is sufficient for us to uniquely construct the topological graph $g$ of $\mathcal{C}$ within $B$. Without loss of generality, we suppose that the curve $\mathcal{C}$ is $x$-regular in $B$. Hence, there is no singularity of $\mathcal{C}$ in $B$. Furthermore, this also guarantees that there is no 'turning-back' of the curve tangent along $x$-direction, so the mapping of $\mathcal{C}$ onto the $x$-axis is injective. Intuitively, the mapped curve should be a series of non-overlapping line segments, whose ends correspond to the intersections between the curve $\mathcal{C}$ and the cell, and such a mapping is injective.

This property leads us to a unique way to connect those intersection points, once they are computed in order to obtain a graph representing the topology of $\mathcal{C}$.

\footnotetext{
Algorithm 4.1 (Connection for a $x$-regular space curve.)

INPUT: a curve $\mathcal{C} x$-regular in a domain $B=\left[a_{0}, b_{0}\right] \times\left[a_{1}, b_{1}\right] \times\left[a_{2}, b_{2}\right]$.

1. Compute the points $p_{1}, \ldots, p_{k}$ of $\mathcal{C} \cap \partial B$.

2. Remove the points on the edges of $B$ for which there is no interior tangent vector of $\mathcal{C}$ to $B$. Duplicate the points where the curve is tangent to a face of $B$ and on the same side of this facet at this point.

3. Sort this set of points by lexicographic order with $x>y>z$.

4. Connect by arcs the pairs of consecutive odd and even index points $\left(\boldsymbol{v}_{2 i-1}, \boldsymbol{v}_{2 i}\right)$, $i=1 \leq \cdots \leq k / 2$, in this sorted list.
} 
OUTPUT: a set of points on the curve and arcs connecting them.

A similar algorithm applies by exchanging $x$ with $y$ or $z$.

Notice that in order to apply this algorithm, we need to compute the points of $\mathcal{C} \cap B$, that is to solve a bivariate system of each facet of $B$. This is performed by applying the algorithm described in Section 2.3.

The special treatment of points of $\mathcal{C}$ on an egde of $B$ or where $\mathcal{C}$ is tangent to a face requires the computation of tangency informations at these points. This is performed by evaluating the derivatives of the defining equations of $\mathcal{C}$ at these points.

\subsection{Algorithm of subdivision}

Collecting these properties, we arrive at the following subdivision algorithm, which subdivides the domain $B$ until some size $\epsilon$, if the curve is not regular in $B$.

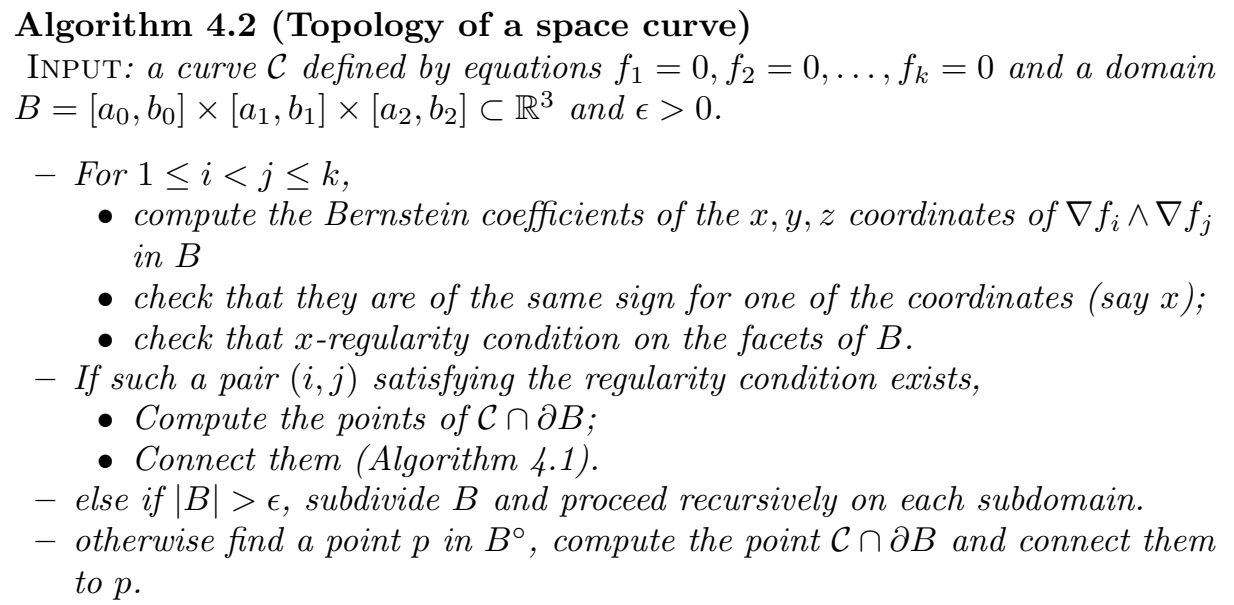

OUTPUT: a set of points $p$ and a set of arcs connecting them.

As in the $2 \mathrm{D}$ case, we have the following "convergence" property:

Proposition 4.3. For $\epsilon>0$ small enough, the graph of points and arcs computed by the algorithm has the same topology as $\mathcal{C} \cap B$.

\section{Surfaces}

In this section, we consider a surface $\mathcal{S}$ defined by the equation $f(x, y, z)=0$, with $f \in \mathbb{Q}[x, y, z]$. We assume that $f$ is squarefree, that is $f$ has no irreducible factors of multiplicity $\geq 2$. The results presented in this section extend those of [2] to the treatment of singular surfaces. However, the complexity analysis provided in [2] has not yet been carried out for such surfaces. 


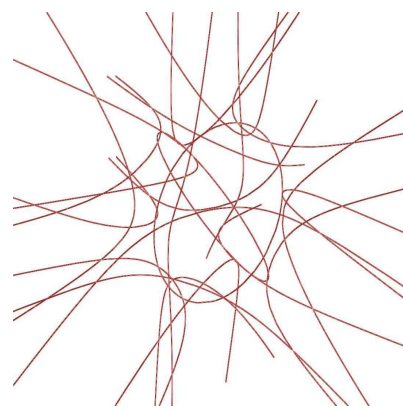

This curve is defined by $f(x, y, z)=0, \partial_{z} f(x, y, z)=0$ where

$$
\begin{aligned}
f(x, y, z)= & 4\left(\tau^{2} x^{2}-y^{2}\right)\left(\tau^{2} y^{2}-z^{2}\right)\left(\tau^{2} z^{2}-x^{2}\right) \\
& -(1+2 \tau)\left(x^{2}+y^{2}+z^{2}-1\right)^{2}, \tau=\frac{1}{2}(1+\sqrt{5}),
\end{aligned}
$$

of degree 6 is defining the surface $S$ called Barth's Sextic. This curve, called the polar curve of $S$ in the $z$ direction, is of degree $30=6 \times 5$. We compute the topology by approximating the coefficients of $f$ and $\partial z f$ by floating point numbers.

Fig. 5. The polar curve of Barth sextic.

Unlike in the 2 dimensional case, the topology of the singular locus and the way to smooth locus is attached to it can be really complicated. To handle such complexity we rely on a technique from singularity theory, namely stratification. For instance, this technique has been fruitfully used in [31] to extend Morse theory to singular varieties. The idea behind stratification is that one can partition a set into submanifolds (the strata) that are connected nicely. Historically, it has been hard to give a formal meaning to "connect nicely". The first idea is that the strata should not be transverse to each other.

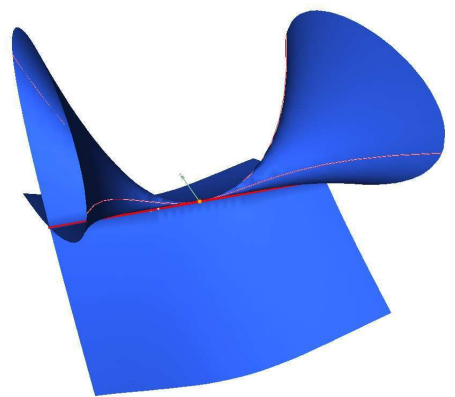

Fig. 6. The double trumpet surface.

Unfortunately this straightforward notion of "nice connection" is not strong enough to ensure good topological properties. A good example is given by the "double trumpet" (see Fig. 6) picture: all the tangent planes connect smoothly to the singular line, nevertheless one would like to tell that the origin of the two trumpets is special. This is achieved by a stronger condition that was defined by H.Whitney in [42] that is now called condition B. 
Definition 5.1 (Whitney's condition B). $\forall M, N$ submanifolds, $\forall x \in M \cap \bar{N}$, we say that $(M, N)$ satisfies condition $B$ at $x$ iff $\forall x_{n} \in M$ converging to $x$, $\forall y_{n} \in N$ also converging to $x$, such that for some line $l$ and tangent space $\tau$ $\lim _{n \rightarrow \infty}\left(x_{n} y_{n}\right)=l$ and $\lim _{n \rightarrow \infty} T_{y_{n}}(N)=\tau$ then $l \subset \tau$ where $\left(x_{n} y_{n}\right)$ denotes the line passing through those points.

The main point that Whitney proved is that for algebraic varieties, "condition B is a stratifying condition". This means that recursively eliminating those points where condition B is not met for the smooth locus, and relegating them to a new subvariety on which we will once again eliminate points not satisfying condition $\mathrm{B}$ in the subvariety, and so on... is a process that actually yields a partition of the variety into submanifolds where all pairs satisfy Whitney's condition B.

Definition 5.2 (Whitney stratification). Let $E$ a set, and $S$ a partition of $E, \mathcal{S}$ is Whitney stratification of $E$ iff

- every stratum $\sigma \in \mathcal{S}$ is a submanifold.

- $\forall \sigma_{1}, \sigma_{2} \in \mathcal{S},\left(\sigma_{1} \cap \overline{\sigma_{2}} \neq \emptyset\right) \Rightarrow \sigma_{1} \subset \overline{\sigma_{2}}$. This is called the boundary condition.

- $\forall K \subset \mathbb{R}^{n}$ compact, $\{\sigma \in \mathcal{S}: \sigma \bigcap K \neq \emptyset\}$ is finite. This is the local finiteness condition.

- $\forall \sigma_{1}, \sigma_{2} \in \mathcal{S}, \forall x \in\left(\sigma_{1} \bigcap \overline{\sigma_{2}}\right),\left(\sigma_{1}, \sigma_{2}\right)$ satisfies condition $B$ at $x$

Actually condition B implies the boundary condition. But because this is not straightforward it is usual to include the boundary condition in the definition as it helps a lot imagining how the different strata are connected together. Another such geometric result is that if $\sigma_{1} \subset \partial \sigma_{2}$ then $\operatorname{dim}\left(\sigma_{1}\right)<\operatorname{dim}\left(\sigma_{2}\right)$.

For surfaces we have an effective way to determine such a Whitney stratification. It is based on a 3d-curve called "polar variety" that is made of the critical points of a linear projection to a plane, in particular it contains the singular locus.

- The 2-dimensional strata is the smooth part of the surface minus the polar curve.

- The 1-dimensional strata is the smooth part of the polar variety.

- The 0-dimensional strata is the singular points of the polar variety.

The proof that this is indeed a Whitney stratification has been carried out in [34] and it is a consequence of a theorem of Speder [39].

The main result that Whitney stratifications yield is topological triviality. Going back to the "double trumpet" case, the topology of the intersection of the surface with a vertical plane changes when the plane reaches the central point. That point is not distinguished by condition A because the trumpet narrows fast, unlike a cone $x^{2}+y^{2}-z^{2}$ for instance. From this simple geometric fact that the topology changes, one can tell that this central point has to be singled out by condition B. This link between Whitney stratifications and topological triviality has been formalized by Thom and is known as Thom's isotopy lemma $([40,32])$. 


\section{Definition 5.3 (Proper stratified submersion).}

Let $f: \mathbb{R}^{n} \rightarrow \mathbb{R}^{m}$, and a set $Z \subset \mathbb{R}^{n}$ Whitney stratified by $\mathcal{S}$, we say that $f$ is a proper stratified submersion for $\mathcal{S}$ iff $\left.f\right|_{Z}$ proper and $\forall \sigma \in \mathcal{S}$, the differential map $\left.\partial f\right|_{\sigma}$ has constant rank $m$.

\section{Theorem 5.1 (Thom's isotopy lemma).}

$\forall Z \subset \mathbb{R}^{n}, \forall \mathcal{S}$ Whitney stratification of $Z, \forall g: \mathbb{R}^{n} \rightarrow \mathbb{R}^{m}$ such that $g$ smooth proper stratified submersion for $\mathcal{S}$,

$\exists h: Z \rightarrow \mathbb{R}^{m} \times\left(g^{-1}(0) \cap Z\right)$ stratum preserving homeomorphism, such that $h$ smooth on each stratum, and $\Pi_{\mathbb{R}^{m}} \circ h=g$.

Now that we have acquired a proper understanding of how an algebraic variety can be decomposed into smooth strata that fit nicely together, we can actually take advantage of this decomposition to determine conditions sufficient to know the topology in small enough boxes. Indeed, as our algorithm proceeds by subdivision, we will finally end up with boxes as small as we want. So we just have to take care of what happens locally. We know we can split the surface into patches of 2-dimensional strata (the smooth part), 1-dimensional strata (singular curves), and 0-dimensional strata (singular points were the 1-dimensional strata do not meet condition B). Topologically we can characterize the topological situation as follows:

- Near a 2-dimensional stratum the topology is the same as a hyperplane.

- Near a 1-dimensional stratum the topology is the same as a cylinder on a singular planar curve.

- Near a 0-dimensional stratum the topology is the same as a cone with center, the isolated point of one of these 0-dimensional stratum and with base, the intersection of the surface with a small sphere and

And we know only one of these three situations can and will happen locally. So we just have to design a solution for each one of the above three cases. For efficiency reasons the criteria we have designed work for situations more general than the limit three cases. The 2-dimensional strata criterion can succeed even with several hyperplanes in the box not only just one. For the 1-dimensional stata we can triangulate even when some patches of the 2-dimensional strata lie in the box even though they are disconnected from the singular locus (in the box). In the case of the 0-dimensional strata we need to have the exact topology in the box. Of course the criteria eventually succeed if the box is small enough. We now describe each one of these criteria and the matching connection algorithm.

\subsection{Regularity criterion for surfaces}

In this section, we consider a domain $B=[a, b] \times[c, d] \times[e, f] \subset \mathbb{R}^{3}$ and a surface $\mathcal{S} \subset B$. The $x$-faces (resp. $y, z$-facet) of $B$ are the planar domains of the boundary $\partial B$ of $B$, orthogonal to the direction $x$ (resp. $y, z$ ).

Definition 5.4. The surface $\mathcal{S}$ is z-regular (resp. $y, z$-regular) in the domain $B$ if, 
- $\mathcal{S}$ has no tangent line parallel to the $z$-direction (reps. $x, y$-direction),

- $\mathcal{S} \cap F$ is regular, for $F$ a $z$-facet (resp. $x, y$-facet) of $B$.

We will say that $\mathcal{S}$ is regular in $B$ if it is regular in $B$ for the direction $x, y$ or $z$. Here again, if a point $p \in \mathcal{S}$ is singular, then any line through this point is tangent to $\mathcal{S}$ at $p$. Thus a surface in $B$ which is regular is also smooth.

Proposition 5.1. If $\mathcal{S}$ is regular, then its topology is uniquely determined by its intersection with the edges of $B$.

Proof. Exchanging the role of $x, y$ or $z$ if necessary, we can assume that $\mathcal{S}$ is $z$-regular in $\mathcal{S}$. As $\mathcal{S}$ has no vertical tangent in $B$, there is no closed connected component of $\mathcal{S}$ in $B$ and each patch of $\mathcal{S} \cap B$ intersects the boundary $\partial B$.

As there is no point of $\mathcal{S}$ with vertical tangent in $B, \mathcal{C}_{F}=\mathcal{S} \cap F$ is $z$-regular for any $x$ or $y$ facet $F$ of $B$. By hypothesis, the curve $\mathcal{C}_{F}=\mathcal{S} \cap F$ is also regular for the $z$-facets of $B$. By proposition 3.1, for any facet $F$ of $B$, the topology of $\mathcal{C}_{F}$ is uniquely determined by the points of $\mathcal{C}_{F} \cap \partial F$. In other words, the topology of $\mathcal{S} \cap \partial B$ is uniquely determined by the intersection of $\mathcal{S}$ with the edges of $B$.

Since $\partial \mathcal{S} \cap B \subset \partial B$, otherwise $\mathcal{S}$ would not be smooth in $B^{\circ}$ or would have a vertical tangent, each patch $\mathcal{P}$ of $\mathcal{S} \cap B$ is simply connected. Moreover, the boundary $\partial \mathcal{P}$ is made of branches of $\mathcal{C}_{F}$ for $F$ a facet of $B$, which share an end point on the edges of $B$. Thus the patches $\mathcal{P}$ of $\S \cap B$ are uniquely determined by the cycles of arcs of $\mathcal{S} \cap \partial B$.

This leads to the following algorithm:

Algorithm 5.1 (Connection of a regular surface)

INPUT: $A$ domain $B \subset \mathbb{R}^{3}$ and a surface $\mathcal{S}$ which is regular in $B$.

- Compute the set $\Xi$ of intersection points of $\mathcal{S}$ with the edges of $B$;

- Deduce the set $\Sigma$ of branches of $\mathcal{S} \cap \partial B$ (using algorithm 3.1);

- Extract the connected components $\mathcal{P}_{1}, \ldots, \mathcal{P}_{k}$ of the graph $\Gamma=(\Xi, \Sigma)$ with vertices $\Xi$ and edges $\Sigma$.

OutPut: The boundary of each patch of $\mathcal{S} \cap B$ described by the cycle of branches $\mathcal{P}_{i}$, for $i=1, \ldots, k$.

Since these arcs form the boundary of $\mathcal{S} \cap B$, locally the part of the surface which is inside $B$ is on "one side" of this arc. This defines a half plane on one side on the tangent line on the tangent plane at a point of such arc.

For a point $p$ on an edge of $B$, which belongs to two arcs, we define the interior tangent sector of $\mathcal{S}$ at $p$ as the intersection of the two corresponding half planes and denote it by $T_{p}^{i}(S)$.

As in the 2D case, simple tests of regularity can be derived from the representation of $f$ in the Bernstein basis.

Lemma 5.1. Let $(u, v, w)$ be any permutation of $(x, y, z)$. Suppose that $\partial_{u} f \neq 0$ in $B$ and that $\mathcal{S} \cap F$ is regular on the u-facets $F$ of $B$. Then $S$ is regular in $B$.

Proof. As $\partial_{u} f \neq 0$ in $B, \mathcal{S}$ has no tangent parallel to the $u$-direction in $B$ and its $u$-facets are regular, so that $\mathcal{S}$ is $u$-regular in $B$. 
Proposition 5.2. Let $(u, v, w)$ be any permutation of $(x, y, z)$. Suppose that the coefficients of $\partial_{u} f$ in the Bernstein basis of $B$ have the same sign $\in\{-1,1\}$ and that the coefficients of $\partial_{v} f$ or $\partial_{w} f$ on the $u$-facets of $B$ are also of the same sign $\in\{-1,1\}$. Then $\mathcal{C}$ is regular in $B$.

Proof. By the convex hull property $\partial_{u} f \neq 0$ in $B$ and $\partial_{v} f \neq 0$ or $\partial_{w} f \neq 0$ on a $u$-facet $F$ of $B$. This implies that the curve $\mathcal{S} \cap F$ is regular in $F$ and therefore that $\mathcal{S}$ is regular in $B$.

This criterion implies that in the valid cells, the derivative of $f$ in one direction is of constant sign and on the two faces transversal to this direction, another derivative is of constant sign. This may be difficult to obtain, when a point of the surface where two derivatives vanish is on (or near) the boundary of the cell. A situation where $\partial_{u} f \neq 0$ but where both derivative $\partial_{v} f, \partial_{w} f$ are not of constant sign on a $u$-facet $F$ of $B$, can be handled by applying recursively the $2 \mathrm{D}$ algorithm of the facet $F$.

\subsection{The polar variety}

As we said before, the way we get a handle on the lower dimensional strata is the polar variety. This object defines a 3d-curve that we will consider to be the closure of our 1-dimensional strata. And we will define the 0-dimensional strata as the singular points of this curve. It was proved in [34] as a consequence of a theorem of Speder [39] that this process yields a Whitney stratification

Now we explain how this object is defined and computed. The polar variety is dependent on a direction that can be chosen arbitrarily. For simplicity, we will consider that this direction is the $z$-direction.

Definition 5.5. The polar variety of $\mathcal{S}$ for the direction $z$, denoted $\mathfrak{C}_{z}(\mathcal{S})$ is the set of points $p \in \mathcal{S}$, such that the line at $p$ of direction $z$ is tangent to $\mathcal{S}$. It is defined by the equations $f(x, y, z)=0, \partial_{z} f(x, y, z)=0$.

Notice that $\mathfrak{C}_{z}(\mathcal{S})$ contains the set of singular points of $\mathcal{S}$ (satisfying $f=\partial_{x} f=$ $\left.\partial_{y} f=\partial_{z} f=0\right)$.

As we assume that $f$ is squarefree, if $\partial_{z} f \neq 0$ then the algebraic variety defined by $f=0, \partial_{z} f=0$ is a curve (ie. of dimension 1 ). However, it can contain irreducible components with multiplicities $\geq 2$, which might induce problems in subdivision techniques.

In order to remove these multiplicities, we need to compute the radical $I\left(\mathfrak{C}_{z}(\mathcal{S})\right)$ of the ideal $I:=\left(f, \partial_{z} f\right)$.

Let us denote by $J(f, g)$ the ideal of $\mathbb{R}[x, y, z]$ generated by the coordinates of $\nabla f \wedge \nabla g$. Recall that for two ideal $I, I^{\prime}$ of $\mathbb{R}[x, y, z],\left(I: I^{\prime}\right)=\{f \in \mathbb{R}[x, y, z] ; \forall g \in$ $\left.I^{\prime}, f g \in I\right\}$. We compute the radical ideal of $I$, as follows:

Proposition 5.3. If $\partial f \neq 0$, the radical $I\left(\mathfrak{C}_{z}(\mathcal{S})\right)$ is $(f, \partial f): J\left(f, \partial_{z} f\right)$.

Proof. It is a direction application of [14].

This ideal division $(I: J(I))$ can be computed by classical algebraic techniques, such as Gröbner bases [10]. We consider this step as a preprocessing step, which 
computes a minimal set of generators $g_{1}, \ldots, g_{k}: I\left(\mathfrak{C}_{z}(\mathcal{S})\right)=\left(g_{1}, \ldots, g_{k}\right)$. Hereafter, we are going to assume that these generators are computed before the subdivision process starts.

We are going to use the topology of the polar curve in order to deduce the topology of the whole surface $\mathcal{S}$. For that purpose, we use algorithm 4.2.

Let us describe now how the topology of the surface is deduced in a domain where the polar curve is $x$-regular (we handle the other direction symmetrically). We will suppose moreover that this polar curve is connected in $B$, that is made of one $x$-regular arc connecting two points on $\mathcal{S} \cap \partial B$ with distinct $x$-coordinates. We also suppose that the topology of $\mathcal{S} \cap \partial B$ is known, that for each facet $F$ a set of arcs of $F$ connecting points on the edges or on the polar curve are known. For that purpose, we apply the algorithm described in section 3. This all happens (in one direction $x$ or $y$ or $z$ ) when we are close enough to the 1-dimensional strata but away from the 0-dimensional strata.

Because the polar curve is $x$-regular we know that the projection to the $x$ axis is a stratified submersion. It is proper because we look at what's in the box. Therefore by Thom's isotopy lemma we know that we have a trivializing homeomorphism, which in this case just deforms the surface in the box to a cylinder over a planar curve (that can be obtained by any intersection of the surface with a $x=$ constant plane).

In order to recover those patches of the surface that connect along the polar curve, we follow the arcs on the facets, starting from a point on the polar variety. The remaining arcs which are not involved in these patches are connected using the algorithm 5.1 for regular surfaces. This leads to the following algorithm:

\section{Algorithm 5.2 (Connection for surface with regular polar variety)}

INPUT: $A$ surface $\mathcal{S}$ and a box $B$ where the polar variety of $\mathcal{S}$ is regular and connected. On each facet, a set of arcs connecting points on the boundary, or on the polar curve, describing the topology of $\mathcal{S} \cap \partial B$.

- Remove the arc of the polar variety from the initial set of arcs and its two points on $\partial B$.

- Compute the connected components of the remaining set of arcs and points.

- For each connected component which starts and ends at the two points of the polar variety, add the polar arc and form the corresponding patch.

- For each of the remaining connected components, which form cycles, build the corresponding patches.

Output: The boundary of each patch of $\mathcal{S} \cap B$ described as a list of cycles of arcs, taken from the input set of arcs.

\subsection{The singular locus of the polar curve}

Finally, we compute the topology of the surface around the 0-dimensional strata, that is the singular points of the curve. Indeed, as we have a radical ideal defining the curve, they can be obtained by looking at the points where all the $2 \times 2$ determinants of the derivatives of the generators of the ideal $I\left(\mathfrak{C}_{z}(\mathcal{S})\right)$ vanish. 
We are now left with those boxes where the polar variety is singular. We can assume that there is only one singular point of the polar variety in the box, and that the variety is close to its tangent cone. Because we are close to the tangent cone, all the tangent lines and planes to the 1 and 2-dimensional strata almost go through the singular point of the polar variety. Therefore they are transverse to the balls containing the singular point and we can apply Thom's isotopy lemma to deduce that the topology in the balls is invariant by radius reduction. Thus the topology in a ball is the same as a cone with center the singular point of the polar curve, and with base the intersection of the surface with the boundary of the ball. For a small enough box $B$, which contains the singular point $p$, the surface will also be like cone with center $p$ and with base $\mathcal{S} \cap \partial B$.

In order to determine the topology of the surface $\mathcal{S}$ in $B$, we compute the topology of $\mathcal{S} \cap \partial B$ using Algorithm 3.2. Then we just connect all points and arcs in the boundary to the central point.

\footnotetext{
Algorithm 5.3 (Topology of a surface)

INPUT: a surface $\mathcal{S}$ defined by a squarefree equation $f(x, y, z)=0$, a domain $B_{0}=\left[a_{0}, b_{0}\right] \times\left[a_{1}, b_{1}\right] \times\left[a_{2}, b_{2}\right] \subset \mathbb{R}^{3}$ and $\epsilon>0$.

- Compute generators $g_{1}, \ldots, g_{k}$ of the ideal $I\left(\mathfrak{C}_{z}(\mathcal{S})\right)=\left(f, \partial_{z} f\right): J\left(f, \partial_{z} f\right)$.

- Compute the Bernstein coefficients of the $f$ and $g_{i}$ in the Bernstein basis of $B:=B_{0}$.

- If $\mathcal{S}$ is regular in $B$, compute its topological structure by Algorithm 5.1.

- Else if the polar variety $\mathfrak{C}_{z}(\mathcal{S})$ is regular and connected in $B$, compute the topological structure of $\mathcal{S} \cap \partial B$ by Algorithm 3.2 on each facet of $B$

- Else if $|B|>\epsilon$, subdivide the box $B$ and proceed recursively on each subdomain.

- Otherwise find a point $p$ in $B^{\circ}$, compute the topological structure of $\mathcal{S} \cap \partial B$ by Algorithm 3.2 and its link over $p$.

OUTPUT: a set of points, arcs and patches and adjacency relations describing the topology of $\mathcal{S} \cap B_{0}$ arcs connecting them.
}

As in the previous case, for $\epsilon>0$ small enough, the output of this algorithm is topologically equivalent to $\mathcal{S} \cap B$.

\section{Conclusion}

We have presented a comprehensive set of algorithms to determine the topology of singular curves both planar and 3-dimensional, as well as singular surfaces. The approach we chose is subdivision, it allows us to handle problems locally and to make use of numerical approximations and still test properties exactly.

Conceptually, the stability needed to be able to do numerical rounding and still get a correct result is achieved through transversality. Indeed, if two objects are transverse, they will still be transverse after a small perturbation. When striving for this goal our enemy is multiplicity. We take care of it by stratifying 


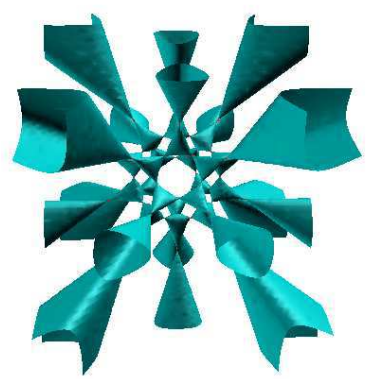

Here is the Barth sextic surface whose polar variety has been computed in and shown in Fig. 5. This surface of degree 6 has the maximun number of isolated singularities for this degree, that is 65. These singular points are also singular points of its polar variety.

Fig. 7. Barth sextic.

the variety and eliminating multiplicity in codimension 1.

Stratifying is easy for curves, but we rely on a theorem of Speder to do this for surfaces. This is the main tumble stone when trying to generalize this method to higher dimensions as then it is not clear how to get a Whitney stratification efficiently.

Getting rid of multiplicity in codimension 1 is nothing more than computing the radical of an ideal. It is easy for principal ideals, this is "taking the squarefree part of the generator". It is trickier to do it efficiently in general, but we managed to do it in our case (when computing the polar variety).

Finally these algorithms still lack a precise assessment of their complexity. Although we know from experience that they are fast. The situation is understood as we can relate the size of the boxes to a measure of transversality. The thorough analysis would have been to heavy for this article as it requires lengthy developments on measures of transversality and bounds on polynomials.

\section{Acknowledgements:}

The topology computation and visualization of this paper have been performed by the softwares AXEL (http://www-sop.inria.fr/galaad/axel/) and SYNAPS (http://www-sop.inria.fr/galaad/synaps/).

This work is partially supported by AIM@Shape (IST Network of Excellence 506766) and ACS (IST FET Open 006413).

\section{References}

1. S.S. Abhyankar. Algebraic Geometry for Scientists and Engineers. American Mathematical Society, Providence, R. I., 1990.

2. L. Alberti, G. Comte, and B. Mourrain. Meshing implicit algebraic surfaces: the smooth case. In L.L. Schumaker M. Maehlen, K. Morken, editor, Mathematical Methods for Curves and Surfaces: Tromso'04, pages 11-26. Nashboro, 2005.

3. J. Gerardo Alcázar and J. Rafael Sendra. Computing the topology of real algebraic space curves. J. Symbolic Comput., 39:719-744, 2005. 
4. J. Bloomenthal. Polygonization of implicit surfaces. Computer-Aided Geometric Design, 5(4):341-355, 1988.

5. J. Bloomenthal. An implicit surface polygonizer. In Paul Heckbert, editor, Graphics Gems IV, pages 324-349. Academic Press, Boston, MA, 1994.

6. J. Bloomenthal. Introduction to implicit surfaces. Morgan Kaufmann, 1997.

7. J.-D. Boissonnat and Oudot.S. Provably good sampling and meshing of surfaces. Graphical Models, 67:405-451, 2005.

8. J.-S. Cheng, X.-S. Gao, and M. Li. Determining the topology of real algebraic surfaces. In Mathematics of Surfaces, number 3604 in LNCS, pages 121-146. SpringerVerlag, 2005.

9. G. E. Collins. Quantifier elimination for real closed fields by cylindrical algebraic decomposition. In Proc. 2nd GI Conference on Automata Theory and Formal Languages, volume 33 of Lecture Notes Comput. Sci., pages 134-183. SpringerVerlag, 1975.

10. D. Cox, J. Little, and D. O'Shea. Ideals, Varieties, and Algorithms: An Introduction to Computational Algebraic Geometry and Commutative Algebra. Undergraduate Texts in Mathematics. Springer Verlag, New York, 1992.

11. T. K. Dey. Curve and Surface Reconstruction: Algorithms with Mathematical Analysis (Cambridge Monographs on Applied and Computational Mathematics). Cambridge University Press, New York, NY, USA, 2006.

12. A. Dickenstein, M.J. Rojas, Rusekz K., Shihx, and J. Extremal real algebraic geometry and a-discriminants. Preprint, 2007.

13. A. Eigenwillig, V. Sharma, and C. K. Yap. Almost tight recursion tree bounds for the descartes method. In ISSAC '06: Proceedings of the 2006 International Symposium on Symbolic and Algebraic Computation, pages 71-78. ACM Press. New York, NY, USA, 2006.

14. D. Eisenbud, C. Huneke, and W. Vasconcelos. Direct methods for primary decomposition. Invent. Math., 110:207-235, 1992.

15. G. Elber and M.-S Kim. Geometric constraint solver using multivariate rational spline functions. In Proc. of 6th ACM Symposium on Solid Modelling and Applications, pages 1-10. ACM Press, 2001.

16. I. Z. Emiris, B. Mourrain, and E. P. Tsigaridas. Real algebraic numbers: Complexity analysis and experimentations. In Reliable Implementation of Real Number Algorithms: Theory and Practice, LNCS. Springer-Verlag, 2007. To appear (also available at http://hal.inria.fr/inria-00071370).

17. G. Farin. Curves and surfaces for computer aided geometric design : a practical guide. Comp. science and sci. computing. Acad. Press, 1990.

18. G. Farin. An ssi bibliography. In Geometry Processing for Design and Manufacturing, pages 205-207. SIAM, Philadelphia, 1992.

19. M. S. Floater. On zero curves of bivariate polynomials. Journal Advances in Computational Mathematics, 5(1):399-415, 1996.

20. E. Fortuna, P. Gianni, P. Parenti, and C. Traverso. Computing the topology of real algebraic surfaces. In ISSAC '02: Proceedings of the 2002 international symposium on Symbolic and algebraic computation, pages 92-100, New York, NY, USA, 2002. ACM Press.

21. G. Gatellier, A. Labrouzy, B. Mourrain, and J.-P. Técourt. Computing the topology of 3-dimensional algebraic curves. In Computational Methods for Algebraic Spline Surfaces, pages 27-44. Springer-Verlag, 2005.

22. L. González-Vega and I. Necula. Efficient topology determination of implicitly defined algebraic plane curves. Comput. Aided Geom. Design, 19(9):719-743, 2002. 
23. T. A. Grandine and F. W. Klein. A new approach to the surface intersection problem. Computer Aided Geometric Design, 14:111-134, 1997.

24. E. Hartmann. A marching method for the triangulation of surfaces. Visual Computer, 14(3):95-108, 1998.

25. J. Hass, R. T. Farouki, C. Y. Han, X. Song, and T. W. Sederberg. Guaranteed Consistency of Surface Intersections and Trimmed Surfaces Using a Coupled Topology Resolution and Domain Decomposition Scheme. Advances in Computational Mathematics, 2007. To appear.

26. D. Kalra and A.H. Barr. Guaranteed ray intersections with implicit surfaces. In Proc. of SIGGRAPH, volume 23, pages 297-306, 1989.

27. J. Keyser, T. Culver, D. Manocha, and S. Krishnan. Efficient and exact manipulation of algebraic points and curves. Computer-Aided Design, 32(11):649-662, 2000 .

28. C. Liang, B. Mourrain, and J.P. Pavone. Subdivision methods for $2 \mathrm{~d}$ and 3d implicit curves. In Computational Methods for Algebraic Spline Surfaces, pages 171-186. Springer-Verlag, 2007. To appear (also available at http://hal.inria.fr/inria-00130216).

29. N. G. Lloyd. Degree theory. Cambridge University Press, Cambridge, 1978. Cambridge Tracts in Mathematics, No. 73.

30. W. Lorensen and H. Cline. Marching cubes: a high resolution 3d surface construction algor ithm. Comput. Graph., 21(4):163-170, 1987.

31. R. MacPherson M. Goresky. Stratified Morse Theory. Springer-Verlag, 1988.

32. J. Mather. Notes on topological stability. Harvard University, 1970.

33. B. Mourrain and J.-P. Pavone. Subdivision methods for solving polynomial equations. Technical Report 5658, INRIA Sophia-Antipolis, 2005.

34. B. Mourrain and J.P.. Técourt. Isotopic meshing of a real algebraic surface. Technical Report 5508, INRIA Sophia-Antipolis, 2005.

35. J.P. Pavone. Auto-intersection de surfaces pamatrées réelles. PhD thesis, Université de Nice Sophia-Antipolis, 2004.

36. T. Sederberg. Algorithm for algebraic curve intersection. Computer Aided Design, 21:547-554, 1989.

37. J.-K. Seong, G. Elber, and M.-S. Kim. Contouring 1- and 2-Manifolds in Arbitrary Dimensions. In SMI'05, pages 218-227, 2005.

38. E. C. Sherbrooke and N. M. Patrikalakis. Computation of the solutions of nonlinear polynomial systems. Comput. Aided Geom. Design, 10(5):379-405, 1993.

39. J. P. Speder. Équisingularité et conditions de Whitney. Amer. J. Math., 97(3), 1975.

40. R. Thom. Ensembles et morphismes stratifiés. Bull. Amer. Math. Soc., 75, 1969.

41. R.J. Walker. Algebraic curves. Springer-Verlag, 1978.

42. H. Whitney. Elementary structure of real algebraic varieties. Annals of Math., 66(2), 1957.

43. A. Witkin and P. Heckbert. Using particles to sample and control implicit surface. In Proc. of SIGGRAPH, pages 269-277, 1994.

44. Y. Zhou, C. Baoquan, and A. Kaufman. Multiresolution tetrahedral framework for visualizing regular volume data. In Proc. of Visualization '97, pages 135-142, 1997. 